\title{
Photoionization and photofragmentation of multiply charged $\mathrm{Lu}_{3} \mathrm{~N} @ \mathrm{C}_{80}$ ions
}

\author{
J. Hellhund, ${ }^{1, *}$ A. Borovik, Jr., ${ }^{1}$ K. Holste,,${ }^{1,2}$ S. Klumpp, ${ }^{3}$ M. Martins, ${ }^{3}$ S. Ricz, ${ }^{1,4}$ S. Schippers,,${ }^{1,2}$ and A. Müller ${ }^{1}$ \\ ${ }^{1}$ Institut für Atom- und Molekülphysik, Justus-Liebig-Universität Gießen, Leihgesterner Weg 217, 35392 Gießen, Germany \\ ${ }^{2}$ I. Physikalisches Institut, Justus-Liebig-Universität Gießen, Heinrich-Buff-Ring 16, 35392 Gießen, Germany \\ ${ }^{3}$ Institut für Experimentalphysik, Universität Hamburg, Luruper Chaussee 149, 22761 Hamburg, Germany \\ ${ }^{4}$ Institute for Nuclear Research of the Hungarian Academy of Sciences (ATOMKI), Bem tér 18/c, H-4026 Debrecen, Hungary
}

(Received 1 June 2015; published 15 July 2015)

\begin{abstract}
Relative cross sections for photoionization of endohedral fullerene ions $\operatorname{Lu}_{3} \mathrm{~N} @ \mathrm{C}_{80}{ }^{q+}(q=1,2,3)$ have been measured employing the photon-ion merged-beam technique. The investigations include various ionization channels $\mathrm{Lu}_{3} \mathrm{~N} @ \mathrm{C}_{80}{ }^{+q \rightarrow+p}(q=1,2,3$ and $p=2,3,4,5,6)$, in some cases accompanied by fragmentation of the carbon cage. Prominent structures related to the carbon $K$-shell ionization threshold were observed in the energy range $280-330 \mathrm{eV}$. These resonance structures have been analyzed in seven product channels and are compared with previously known absorption spectra of several fullerene species. Within the statistical significance of the data, we do not observe any signatures of the $\mathrm{N} 1 s$ and Lu $3 d$ thresholds in the energy ranges 390-435 eV and $1500-1700 \mathrm{eV}$, respectively. By comparing the energies for double ionization of $\mathrm{Lu}_{3} \mathrm{~N} @ \mathrm{C}_{80}{ }^{+}$and $\mathrm{Lu}_{3} \mathrm{~N} @ \mathrm{C}_{80}{ }^{2+}$ at the carbon $1 s K$-shell threshold, we derive a value of $5.0 \pm 0.4 \AA$ for the radius of the carbon cage.
\end{abstract}

DOI: 10.1103/PhysRevA.92.013413

PACS number(s): 33.80.Eh, 33.70.Fd, 81.05.ub

\section{INTRODUCTION}

Endohedral fullerenes are nanoscale systems that have received much attention in the last decade. Numerous ideas for applications ranging from medical usage [1-4] (such as in imaging or drug delivery) to their employment in devices for quantum computing [5] and the possibility of finding novel fundamental effects $[6,7]$ make their examination a focus of research.

Shortly after Kroto et al. had discovered fullerenes in 1985 [8], they found indications suggesting the existence of the endohedral compound La@ $\mathrm{C}_{60}$ [9]. The existence of such compounds has been confirmed by Kroto et al. several years later [10]. This finding has stimulated intense theoretical research in the field of endohedral fullerenes. One direction of this research has addressed the screening effects of the fullerene cage on the electron or photon emission of an encapsulated atom [7,11-20]. The common prediction of these screening models is a redistribution of photoionization resonance strengths from the cage to the encapsulated atom or vice versa.

In spite of the increasing number of theoretical studies, there are relatively few measurements. The limited access to endohedral substances impeded experimental studies. First experimental results on photoionization of neutral metallofullerenes in the gas phase were obtained by Mitsuke et al. [21,22] in 2005. In photoionization experiments with Ce@ $\mathrm{C}_{82}{ }^{+}$and $\mathrm{C}_{82}{ }^{+}$ions, a Gießen-Reno-Berkeley collaboration could demonstrate the excess cross section due to the presence of the encapsulated $\mathrm{Ce}$ atom compared to the empty-shell fullerene [23]. A weaker excess amplitude was found for $\mathrm{Sc}_{3} \mathrm{~N} @ \mathrm{C}_{80}$ [24-26]. The ongoing experimental progress in the field of endohedral fullerenes led to intensified theoretical efforts. In particular, confinement resonances in the photoionization of endohedral atoms were already predicted in 1993 [6] and intensely discussed but even their very existence

*jonas.hellhund@iamp.physik.uni-giessen.de was questioned again in 2010 [27]. Later in 2010, a first answer to that question was provided by Kilcoyne et $\mathrm{al}$. who presented experimental data suggesting that the excess cross section originating from the encapsulated xenon atom was modulated due to the atom's confinement within the fullerene cage [28]. In 2013, this result was consolidated by Phaneuf et al. [29] who measured photoionization cross sections of $\mathrm{Xe} @ \mathrm{C}_{60}{ }^{+}$ with unprecedentedly low statistical uncertainty.

Characteristic signatures of specific elements in endohedral fullerenes and their modification by the chemical and physical environment are also the topic of the present experimental work. The choice of the specific endohedral fullerene that has been used as a target for the present photoionization experiments was influenced by the availability of target material and the energy range of the synchrotron light source where the experiments could be conducted. The measurements were carried out using the permanent end station PIPE (Photon-Ion spectrometer at PETRAIII) [30] at the Variable Polarization XUV Beamline P04 [31] of the synchrotron PETRA III at DESY in Hamburg. At the time of the present experiment, photons with energies between $250 \mathrm{eV}$ and (at the time of the experiment) about $2000 \mathrm{eV}$ were available at this beamline. These energies are considerably larger than those that were previously available for photon-ion merged-beam experiments. Consequently, the scope of the current effort was to extend our recent studies on photoionization of fullerenes [32-34] as well as endohedral fullerenes [23-26,28,29,35] to heavier species. Here, we present cross sections for photoionization and photofragmentation of endohedral fullerene ions $\mathrm{Lu}_{3} \mathrm{~N} @ \mathrm{C}_{80}{ }^{q+}(q=1,2,3)$.

Characteristic features of atoms in the photoionization cross sections were expected in the vicinity of subshell ionization edges and these had to be matched with the accessible photon energies. In particular, the carbon $K$ edge near $295 \mathrm{eV}$, the nitrogen $K$ edge near $410 \mathrm{eV}$, and some lutetium $M$ edges above $1500 \mathrm{eV}$ are in the accessible photon energy range. The present measurements concentrated mainly on the energy range of the carbon $K$ edge. Central questions of the present investigation concern the mutual influence between carbon 
cage and encapsulated molecular compound. How are the characteristic features of the encapsulated atoms affected by a surrounding fullerene cage and, vice versa, how is the response of the carbon cage affected by the presence of encapsulated atoms or molecules?

The layout of this paper is as follows: Section II details the experimental setup. Section III presents and discusses the results obtained from the experimental work. Finally Sec. IV provides a summary and conclusions.

\section{EXPERIMENTAL SETUP}

\section{A. Ion beam preparation}

Few species of endohedral fullerenes are commercially available today. Restrictions are given by the stability and the availability of a suitable process for the synthesis of a desired species. A further complication is purification. For the present study, we used a commercially available sample of endohedral $\mathrm{Lu}_{3} \mathrm{~N} @ \mathrm{C}_{80}$ fullerene powder with a purity of $35 \%$. Complete purification was achieved by in situ mass separation (see below).

The nanoscale system $\mathrm{Lu}_{3} \mathrm{~N} @ \mathrm{C}_{80}$ consists of a planar trigonal molecular compound, $\mathrm{Lu}_{3} \mathrm{~N}$, encapsulated inside a $\mathrm{C}_{80}$ carbon cage. Ross et al. [36] claim that both $\mathrm{Lu}_{3} \mathrm{~N}$ and $\mathrm{C}_{80}$ are unstable concluding that they cannot exist individually. In the case of $\mathrm{C}_{80}$ this is in contrast to the findings of Hennrich et al. [37]. These authors reported the isolation and purification of $\mathrm{C}_{80}$ and subsequent photoemission measurements using a solid $\mathrm{C}_{80}$ sample accompanied by a detailed investigation of the symmetry of $\mathrm{C}_{80}$. Disregarding this controversy, one can state that $\mathrm{Lu}_{3} \mathrm{~N} @ \mathrm{C}_{80}$ is far more stable than $\mathrm{C}_{80}$ because the additional molecule inside stabilizes the surrounding nearly spherical carbon cage from within [36].

For the preparation of the $\mathrm{Lu}_{3} \mathrm{~N} @ \mathrm{C}_{80} q+$ ion beams, the sample material was introduced into the ion source as a solid powder inside a small stainless-steel crucible. This crucible was inserted into a resistively heated oven which can reach temperatures above $1500^{\circ} \mathrm{C}$ at a heating power of about $35 \mathrm{~W}$. The vapor that was generated from the sample powder was ionized by electron bombardment inside a permanent-magnet electron-cyclotron-resonance (ECR) source [38] operated at a potential of $+6 \mathrm{kV}$. After extraction and acceleration of the ions, the ion beam was focused and steered by a series of electrostatic lenses and deflectors. A double-focusing dipole (analyzer) magnet in combination with two four-jaw slits located in front of and behind of the magnet was used for the mass-over-charge selection of the primary ion species to be investigated.

A typical experimental run started with inserting a crucible loaded with sample material (containing 35\% of $\mathrm{Lu}_{3} \mathrm{~N} @ \mathrm{C}_{80}$ and $65 \%$ of other fullerenes, mainly $\mathrm{C}_{60}$ ) into the source. In the first phase after the source chamber had reached operating pressure (better than $10^{-5}$ mbar), the sample was heated with a low power $(2.7 \mathrm{~W})$ such that the crucible remained well below the sublimation temperature of $\mathrm{C}_{60}$. This phase of constant low-power heating took several days during which unwanted volatile contaminants were removed from the sample material while leaving the endohedral component of the sample largely untouched. During this phase, a continuous ECR plasma discharge of argon as carrier gas was sustained to warm up and clean the chamber walls of the source as well as to condition the source for measurement operation. During this conditioning phase, the source chamber reached a base pressure of some $10^{-7}$ mbar.

In a second phase of source operation, the oven power was slowly increased and first weak beams of $\mathrm{C}_{60}{ }^{+}$(which evaporates at lower oven temperatures than $\mathrm{Lu}_{3} \mathrm{~N} @ \mathrm{C}_{80}$ ) were extracted. The characteristic operating pressure inside the ion source then was in the lower $10^{-6}$ mbar region. The aim of this second phase was to obtain an ion beam with high intensity and narrow beam profile while maintaining long-term stability. Optimization of conditions for the extraction of the desired primary ion focused on both tuning the source conditions and the beam optics.

In all phases of operation, mass spectrometry was an important tool for monitoring the ion beam composition. Mass spectra of primary ions were obtained by measuring the primary ion current collected in a Faraday cup directly behind the analyzer magnet as a function of magnetic field strength.

The mass spectrum shown in Fig. 1(a) was recorded after 50 hours of low-power outgassing and 12 hours of carefully powering up the source. At this time, with about $5 \mathrm{~W}$ heating power, the spectrum was dominated by a plethora of peaks from pristine light fullerene ions $\left(\mathrm{C}_{60}, \mathrm{C}_{70}\right)$ and their fragments $\left(\mathrm{C}_{58}, \mathrm{C}_{56}, \ldots\right)$. The current of $\mathrm{C}_{60}{ }^{+}$ions peaked at $160 \mathrm{pA}$. Under the specific conditions during this phase of ion-source operation, $\mathrm{C}_{60}{ }^{+}$was about 30 times more abundant than $\mathrm{Lu}_{3} \mathrm{~N} @ \mathrm{C}_{80}{ }^{+}$although the sample contained about 35\% of $\mathrm{Lu}_{3} \mathrm{~N} @ \mathrm{C}_{80}$ and probably about $50 \%$ of $\mathrm{C}_{60}$. Over time, the balance shifted towards higher masses when the lighter molecules had been evaporated selectively off the sample and the oven temperature was increased further.

Figure 1(b) depicts a mass spectrum from the same sample but 48 hours later after a further continuous increase of the oven power to $12.75 \mathrm{~W}$. Now $\mathrm{Lu}_{3} \mathrm{~N} @ \mathrm{C}_{80}{ }^{+}$dominated the spectrum with an ion current of about $220 \mathrm{pA}$. The second-strongest peak originated from doubly charged $\mathrm{Lu}_{3} \mathrm{~N} @ \mathrm{C}_{80}{ }^{2+}$ ions. Higher charge states, i.e., $3+$ and $4+$, were also found (not shown in Fig. 1).

In addition to the various fragments and charge states of $\mathrm{Lu}_{3} \mathrm{~N} @ \mathrm{C}_{80}$, there are also peaks matching $m / q$ of $\mathrm{Lu}_{2} \mathrm{~N} @ \mathrm{C}_{80}{ }^{+}$and $\mathrm{Lu}_{2} \mathrm{~N} @ \mathrm{C}_{80}{ }^{2+}$ but at this time it remains unclear whether $\mathrm{Lu}_{2} \mathrm{~N} @ \mathrm{C}_{80}$ exists in the initial powder or is formed in the plasma. Obviously $\mathrm{Lu}_{2} \mathrm{~N} @ \mathrm{C}_{80}{ }^{+}$and $\mathrm{Lu}_{2} \mathrm{~N} @ \mathrm{C}_{80}{ }^{2+}$ are sufficiently stable to survive the harsh plasma environment in the source as well as the flight time to their detection at the back end of the apparatus. Furthermore, it is remarkable that the sample seemed not to contain any intact empty $\mathrm{C}_{80}$. The traces at mass $960 \mathrm{u}$ that are present in Fig. 1(a) can be explained as fragments of $\mathrm{C}_{82}$ and $\mathrm{C}_{84}$ based upon the intensity pattern of fullerene breakup [29]. This supports the above mentioned speculation that the empty $\mathrm{C}_{80}$ molecule is unstable but the presence of $\mathrm{Lu}_{3} \mathrm{~N}$ inside does strongly stabilize the compound as suggested by Ross et al. [36].

Behind the mass-over-charge analyzing magnet, the ion beam passed through further steering and focusing elements including an electrostatic quadrupole lens. It was then directed onto the photon axis by a cylindrical electrostatic deflector. 


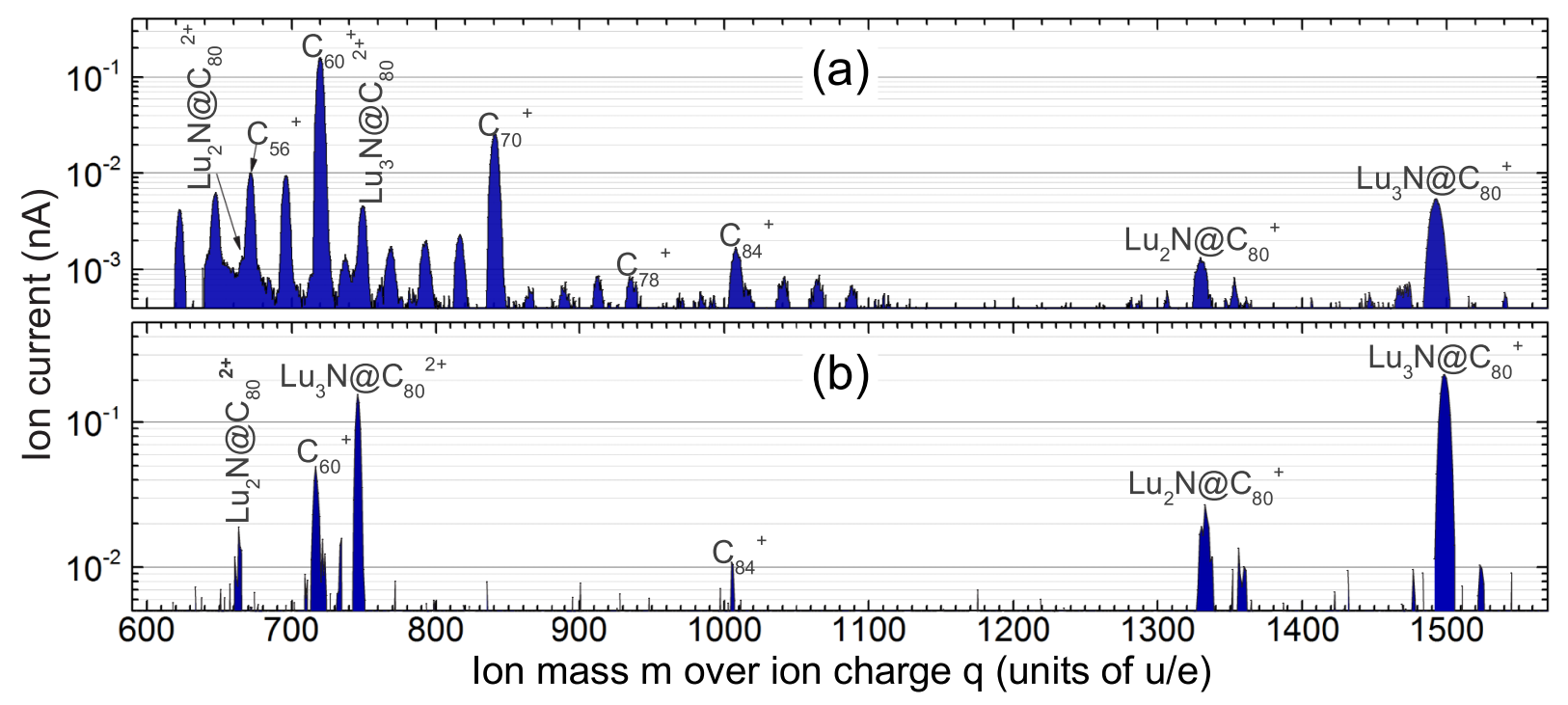

FIG. 1. (Color online) Two primary-ion mass spectra of the same sample at different times: (a) recorded after $50 \mathrm{~h}$ of outgassing followed by $12 \mathrm{~h}$ of slowly increasing the heating power of the oven to $5 \mathrm{~W}$; (b) recorded $48 \mathrm{~h}$ after (a) at a heating power of $12.75 \mathrm{~W}$. The mass-resolving power was 200.

In the interaction region, currents of up to $210 \mathrm{pA}$ of singly charged, $20 \mathrm{pA}$ of doubly charged, and $18 \mathrm{pA}$ of triply charged $\mathrm{Lu}_{3} \mathrm{~N} @ \mathrm{C}_{80}$ primary ions could be made available.

\section{B. Photon-ion interaction}

The interaction between photons and $\mathrm{Lu}_{3} \mathrm{~N} @ \mathrm{C}_{80}{ }^{q+}$ ions led to ionization and fragmentation of the primary ions. Relative cross sections $\sigma(E)$ for these processes were measured by recording the $\mathrm{Lu}_{3} \mathrm{~N} @ \mathrm{C}_{80-x}{ }^{q+}$ production rate as a function of photon energy. The procedures detailed in Ref. [30] were employed. The PIPE setup allows for the determination of absolute cross sections. However, for the present measurements, the beam-overlap factors which are required for obtaining absolute cross sections were not determined because of time constraints. Nevertheless, different product-ion yield spectra are still on the same relative cross section scale and can, therefore, be compared to one another quantitatively since the beam overlap is independent of the product channel investigated. Moreover, the beam overlap can safely be assumed to be independent of photon energy over the narrow photon energy ranges that were scanned within the present study. Consequently, the distribution of oscillator strength among the different product channels can be extracted from the measured spectra although they are not on an absolute scale.

For the photon-ion interaction studies, the photon flux was measured with a calibrated photodiode. It ranged from $10^{12} \mathrm{~s}^{-1}$ up to $10^{13} \mathrm{~s}^{-1}$ depending on photon energy and the selected resolving power. In order to obtain relative cross sections from the measured ion yields, these were normalized on the simultaneously recorded photon flux and primary ion current.

The various reaction products were separated by a second mass-over-charge analyzing dipole magnet (demerging magnet) following the photon-ion interaction region. Prior to the selection of specific reaction products, an overview over the product beam composition was obtained by simul- taneously scanning the second magnet's field strength and recording the product count rate employing a single-particle detector $[39,40]$.

Figure 2 shows the product-ion spectrum resulting from the interaction of $\mathrm{a} \mathrm{Lu}_{3} \mathrm{~N} @ \mathrm{C}_{80}{ }^{+}$beam with $300-\mathrm{eV}$ photons. Various fragment ions and different charge states of $\mathrm{Lu}_{3} \mathrm{~N} @ \mathrm{C}_{80}$ can be identified. Two classes of peaks with different widths can be distinguished: There are few narrow peaks, whereas the vast majority of peaks are broader. The narrow peaks originated from photoreactions resulting in $\mathrm{Lu}_{3} \mathrm{~N} @ \mathrm{C}_{80}{ }^{2+, 3+, 4+}$ and fragments. This was verified by blocking the photon beam upstream of the interaction region: The narrow peaks vanished. A consistent mass calibration was obtained from the three photoion species present. The broad peaks originate from collisions of the primary ions with residual gas particles along their flight path. There are three contributions to the widths of the wider peaks: First, collisions with residual gas can also happen off the photon axis, thus allowing products originating from a larger spatial region to contribute to the detector signal. Second, inelastic collisions (and elastic collisions as well) involve an exchange of momentum resulting in a momentum spread. Third, fragmentation of molecules is accompanied by an internal energy transfer resulting in a kinetic energy release (KER). The distribution of broad peaks follows a periodic pattern where one peak corresponds to a fragment with one carbon dimer more (or less, respectively) than the neighboring peak. We can unambiguously assign mass-over-charge ratios to these peaks which correspond to fragments of $\mathrm{Lu}_{3} \mathrm{~N} @ \mathrm{C}_{80}$ primary particles.

For the present photoionization measurements, the energy resolution was varied between 100 and $220 \mathrm{meV}$ with an uncertainty of $\pm 20 \mathrm{meV}$ at a photon energy $E_{\gamma}=300 \mathrm{eV}$. Investigations showed that varying the resolution has no influence on the beam overlap in this energy region. The photon energy scale for the cross-section measurements described below was calibrated by first applying a Doppler correction to take care of the ion-velocity-related energy shift. 


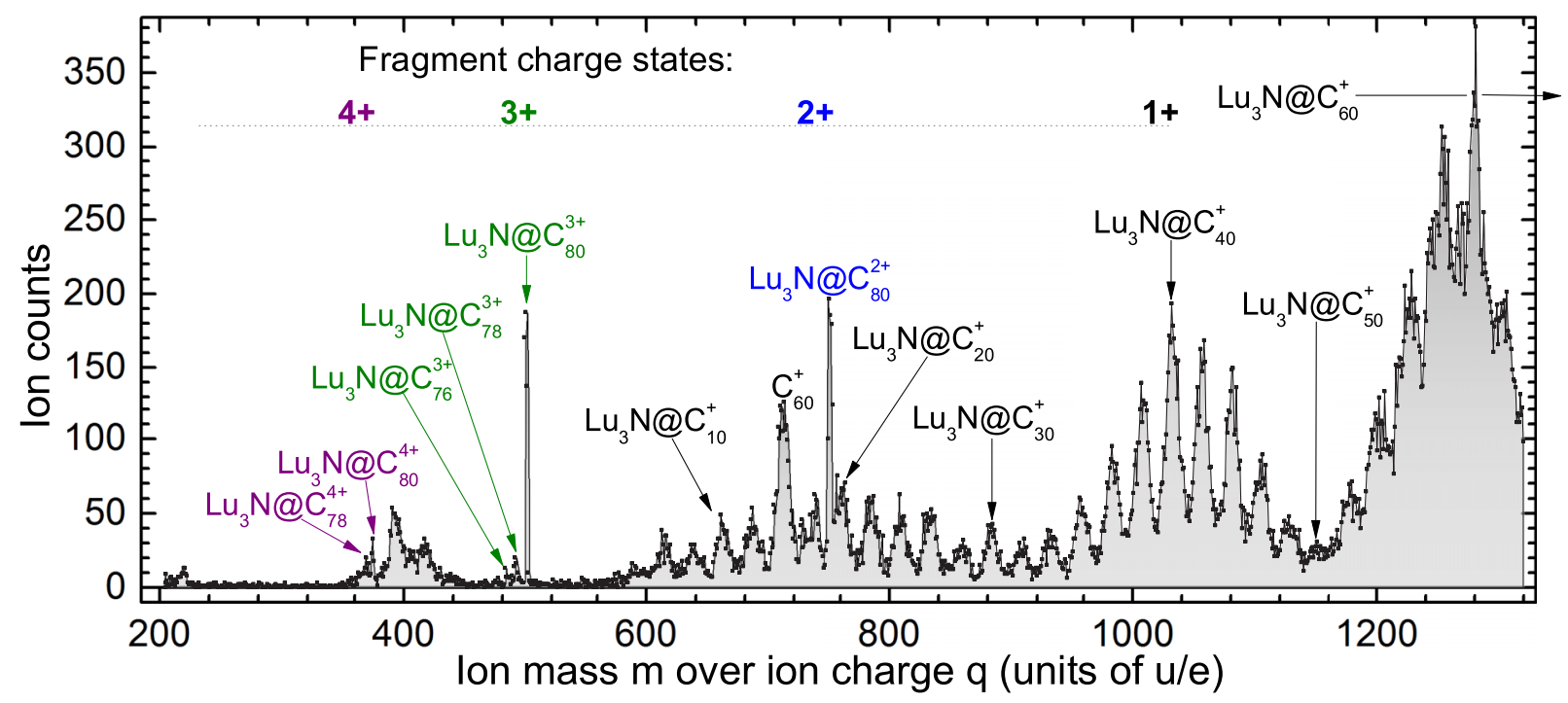

FIG. 2. (Color online) Product-ion mass spectrum obtained by sweeping the demerging magnet field while the photon energy remained at $300 \mathrm{eV}$. The mass scan could not be extended to include the primary $\mathrm{Lu}_{3} \mathrm{~N} @ \mathrm{C}_{80}+$ beam because its intensity would have saturated the single-particle detector.

Thereafter, the monochromator calibration was determined by separately measuring known resonance energies of $\mathrm{C}^{q+}$ and $\mathrm{Ne}^{+}$that were compared with literature values [41-43]. The resulting systematic uncertainty of the present energy scale is $\pm 0.05 \mathrm{eV}$.

\section{RESULTS}

We present relative cross sections for 11 different photoreaction channels of $\mathrm{Lu}_{3} \mathrm{~N} @ \mathrm{C}_{80}{ }^{q+}$ ions at energies around the carbon $K$-shell threshold (280-330 eV) and the lutetium $M$-shell threshold (1500-1700 eV). In particular, we measured relative cross sections for the production of the following heavy fragments (right) from the indicated primary particles (left):

$$
\begin{aligned}
& \mathrm{Lu}_{3} \mathrm{~N} @ \mathrm{C}_{80}{ }^{+} \rightarrow \mathrm{Lu}_{3} \mathrm{~N} @ \mathrm{C}_{80}{ }^{2+}, \\
& \mathrm{Lu}_{3} \mathrm{~N} @ \mathrm{C}_{80}{ }^{+} \rightarrow \mathrm{Lu}_{3} \mathrm{~N} @ \mathrm{C}_{80}{ }^{3+}, \\
& \mathrm{Lu}_{3} \mathrm{~N} @ \mathrm{C}_{80}{ }^{+} \rightarrow \mathrm{Lu}_{3} \mathrm{~N} @ \mathrm{C}_{78}{ }^{3+}, \\
& \mathrm{Lu}_{3} \mathrm{~N} @ \mathrm{C}_{80}{ }^{+} \rightarrow \mathrm{Lu}_{3} \mathrm{~N} @ \mathrm{C}_{80}{ }^{4+}, \\
& \mathrm{Lu}_{3} \mathrm{~N} @ \mathrm{C}_{80}{ }^{+} \rightarrow \mathrm{Lu}_{3} \mathrm{~N} @ \mathrm{C}_{80}{ }^{5+}, \\
& \mathrm{Lu}_{3} \mathrm{~N} @ \mathrm{C}_{80}{ }^{+} \rightarrow \mathrm{Lu}_{3} \mathrm{~N} @ \mathrm{C}_{72}{ }^{5+}, \\
& \mathrm{Lu}_{3} \mathrm{~N} @ \mathrm{C}_{80}{ }^{+} \rightarrow \mathrm{Lu}_{3} \mathrm{~N} @ \mathrm{C}_{80}{ }^{6+}, \\
& \mathrm{Lu}_{3} \mathrm{~N} @ \mathrm{C}_{80}{ }^{+} \rightarrow \mathrm{Lu}_{3} \mathrm{~N} @ \mathrm{C}_{74}{ }^{6+}, \\
& \mathrm{Lu}_{3} \mathrm{~N} @ \mathrm{C}_{80}{ }^{2+} \rightarrow \mathrm{Lu}_{3} \mathrm{~N} @ \mathrm{C}_{80}{ }^{3+}, \\
& \mathrm{Lu}_{3} \mathrm{~N} @ \mathrm{C}_{80}{ }^{2+} \rightarrow \mathrm{Lu}_{3} \mathrm{~N} @ \mathrm{C}_{80}{ }^{4+}, \\
& \mathrm{Lu}_{3} \mathrm{~N} @ \mathrm{C}_{80}{ }^{3+} \rightarrow \mathrm{Lu}_{3} \mathrm{~N} @ \mathrm{C}_{80}{ }^{4+} .
\end{aligned}
$$

\section{A. Results for photon energies 280 to $330 \mathrm{eV}$}

In this section we present relative cross sections for the seven reaction channels [Eqs. (1a)-(1d), (1i), (1j), and (1k)] that have been investigated in the photon energy range
280-330 eV which comprises the carbon $K$-shell threshold. Figure 3 summarizes the results for singly charged primary ions [Eqs. (1a), (1b), and (1d)]. Fig 4 shows data obtained with doubly and triply charged primary ions [Eqs. (1i), (1j), and (1k)] as well as a fragmentation channel [Eq. (1c)].

For the purpose of relative calibration of the spectra, the photoreaction count rates at a single photon energy have been measured in the various reaction channels within a short period of time during which the experimental conditions did not change. As detailed in Sec. II, it is a crucial requirement for determining relative cross sections with this method that the beam overlaps are the same in the different reaction channels. For different product channels of the same primary ion the overlap is the same. This was exploited by all but one of the spectra measured in this study: Only for the cross section scale of the spectrum shown in panel (b) of Fig. 4 do we have to admit a bigger uncertainty of (conservatively) up to $\pm 50 \%$ because this channel's intensity was not recorded during the calibration measurements in which the count rates were determined for each reaction channel at a fixed photon energy. Table I lists the signal and background count rates obtained for the relative scaling.

Additionally, the count rates associated with the unwanted generation of product particles in collisions of primary ions with residual gas particles were determined by independent measurements for each product channel individually and the data have been corrected for this background. Signal-tobackground ratios range from 4:1 up to 65:1. Additionally, the robustness of spectral features against variations in background determination was thoroughly investigated. We estimate an uncertainty of less than $\pm 20 \%$ for the determined background intensity. Since the background is small in comparison to the signal, this background uncertainty does not significantly contribute to the uncertainty of the measured relative cross sections.

A common set of resonances with individual widths and energies seems to make up for the most prominent structures 


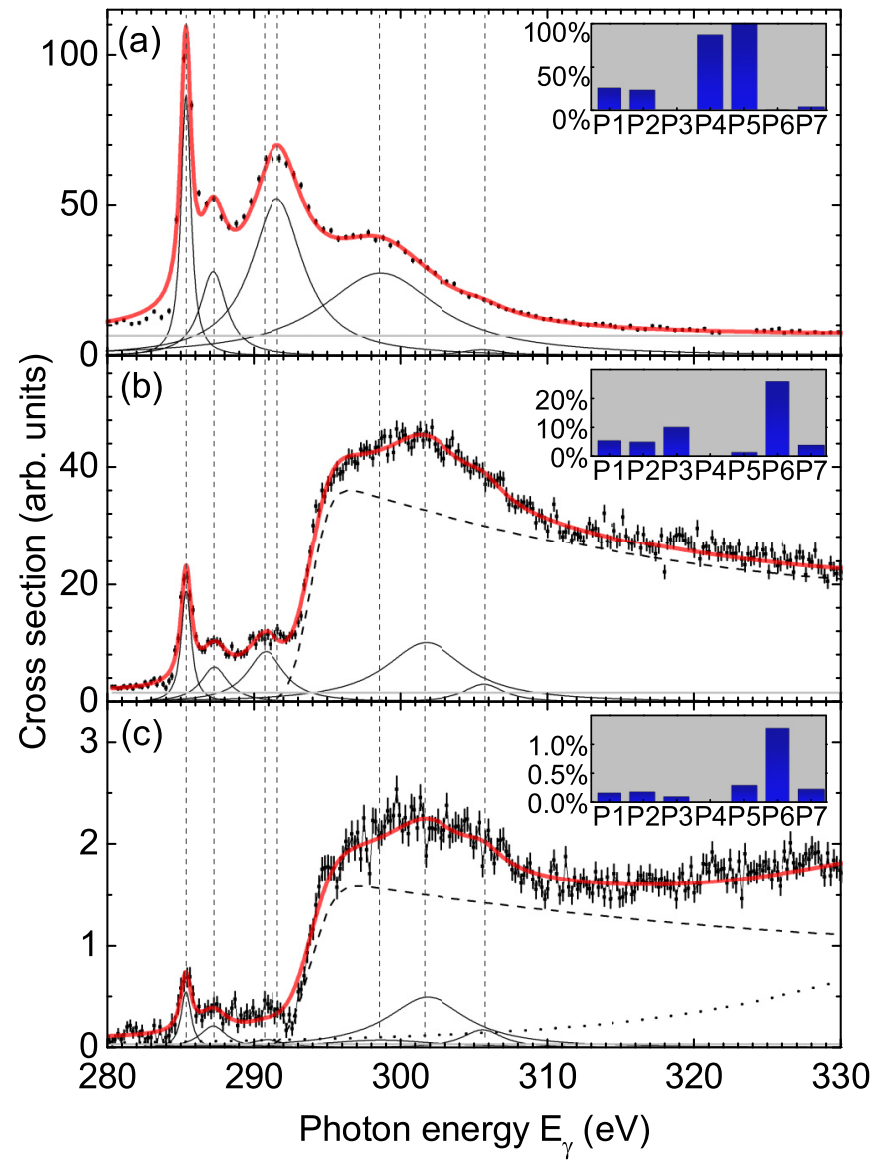

FIG. 3. (Color online) Relative cross sections for single (a), double (b), and triple (c) photoionization of $\mathrm{Lu}_{3} \mathrm{~N} @ \mathrm{C}_{80}{ }^{+}$primary ions. Black square symbols with error bars represent the experimental data. Thick red lines are fitting curves composed of seven Voigt peak profiles (fine solid black lines), a constant background (fine light gray line), and a second order polynomial convoluted with a Gaussian (dashed black line) to model the $K$-shell threshold behavior. In the case of triple ionization, a wide eighth peak was needed for the modeling (dotted gray line). Vertical dashed lines illustrate the peak positions. We observe the same peak positions in the different product channels. The insets show the relative contributions of each resonance area. The largest peak area is normalized to $100 \%$ (see Table II).

in all spectra with the first and most outstanding resonance occurring at $285 \mathrm{eV}$. Therefore, we made an attempt to model each spectrum of product ions with a common set of basis functions.

\section{B. Fitting procedure}

The product-ion spectra were modeled as a sum of seven peak features (Voigt profile resonances, fine solid black lines in Fig. 3; see Table II), a threshold feature (second order polynomial multiplied with a step function and convoluted with a Gaussian, dashed line), and a constant background modeling the carbon $L$-shell contribution to the measured spectra (gray line). The relative strengths of the resonance peaks $\mathrm{P} 1-\mathrm{P} 7$ found in each spectrum are given by the vertical blue bars in the insets. In each inset the peak areas are normalized to the area of the strongest peak (P5) in Fig. 3(a).

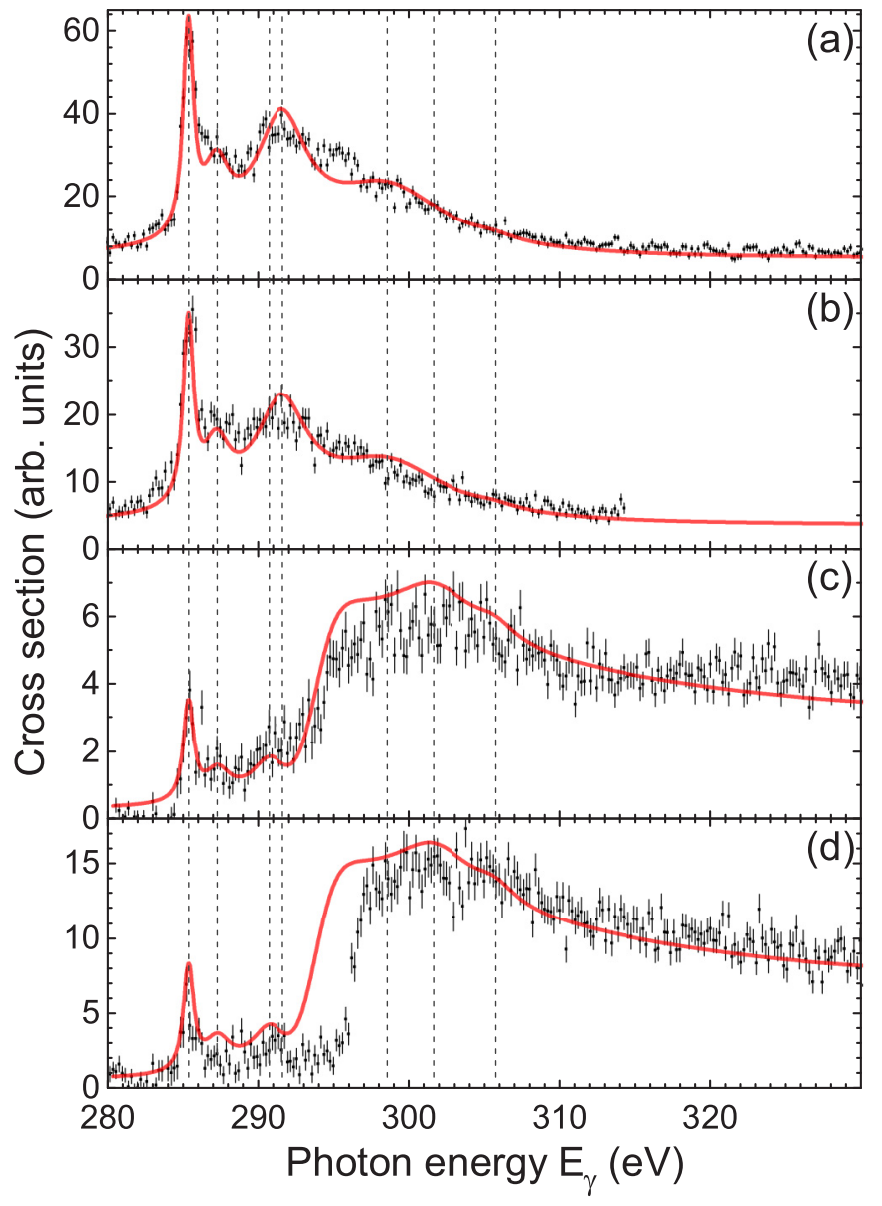

FIG. 4. (Color online) Relative cross sections of photoreactions for singly, doubly, and triply charged $\mathrm{Lu}_{3} \mathrm{~N} @ \mathrm{C}_{80}$ ions. (a) $\mathrm{Lu}_{3} \mathrm{~N} @ \mathrm{C}_{80}{ }^{2+} \rightarrow \mathrm{Lu}_{3} \mathrm{~N} @ \mathrm{C}_{80}{ }^{3+}$ and (b) $\mathrm{Lu}_{3} \mathrm{~N} @ \mathrm{C}_{80}{ }^{3+} \rightarrow$ $\mathrm{Lu}_{3} \mathrm{~N} @ \mathrm{C}_{80} 4+$ both accompanied by the scaled fitting result from Fig. 3(a); (c) $\mathrm{Lu}_{3} \mathrm{~N} @ \mathrm{C}_{80}+\rightarrow \mathrm{Lu}_{3} \mathrm{~N} @ \mathrm{C}_{78}{ }^{3+}$ and (d) $\mathrm{Lu}_{3} \mathrm{~N} @ \mathrm{C}_{80}{ }^{2+} \rightarrow \mathrm{Lu}_{3} \mathrm{~N} @ \mathrm{C}_{80}{ }^{4+}$ both accompanied by the individually scaled fitting results from Fig. 3(b).

In an iterative process, peak positions and natural widths were determined from the respective channels of their most prominent appearance. The parameters for P4 and P5 were determined from Fig. 3(a) data and the parameters for the other resonance features were determined from Fig. 3(b)

TABLE I. Signal and background determination: Counts for signal plus background $(\mathrm{s}+\mathrm{b})$ and background $(\mathrm{b})$ in different product channels for a period of $100 \mathrm{~s}$. A fragmentation channel is denoted by **. $\mathrm{I}_{s+b}{ }^{+}$and $\mathrm{I}_{b}{ }^{+}$denote the primary ion current in units of $\mathrm{pA}$ at the time of the measurement for signal plus background and background only, respectively. The photon energy $E_{\gamma}$ is given in units of $\mathrm{eV}$.

\begin{tabular}{lrrrrrc}
\hline \hline & $\mathrm{I}_{s+b}{ }^{+}$ & $\mathrm{s}+\mathrm{b}$ & $\mathrm{I}_{b}{ }^{+}$ & $\mathrm{b}$ & $E_{\gamma}$ & $\mathrm{s} / \mathrm{b}$ \\
\hline $1+\rightarrow 2+$ & 7.8 & 1594 & 7.6 & 176 & 292 & 7.84 \\
$* * 1+\rightarrow 2+$ & 2.5 & 68 & 2.8 & 12 & 300 & 4.85 \\
$1+\rightarrow 3+$ & 5.9 & 1074 & 4.8 & 13 & 292 & 65.7 \\
$1+\rightarrow 4+$ & 2.5 & 80 & 2.9 & 16 & 300 & 5.33 \\
$2+\rightarrow 3+$ & 9.8 & 1102 & 9.3 & 137 & 292 & 6.58 \\
$2+\rightarrow 4+$ & 13.7 & 930 & 12.6 & 77 & 301 & 10.1 \\
\hline \hline
\end{tabular}


TABLE II. Properties of the group of resonances used for fitting spectra of the seven investigated channels around the carbon $K$-threshold energy as shown in Figs. 3 and 4. The Lorentzian widths of the peaks marked with an asterisk were determined from a single reaction channel, whereas in all other cases the peaks were prominent in two or three channels and therefore their widths could be obtained by simultaneously fitting the results obtained for more than one channel. Columns $A_{\text {s.i. }}, A_{\text {d.i. }}$, and $A_{\text {t.i. }}$ list relations of the peak areas in the single-ionization [s.i., Fig. 3(a)], double-ionization [d.i., Fig. 3(b)], and triple-ionization [t.i., Fig. 3(c)] reaction channels, respectively. The area of the strongest peak was normalized to $100 \%$.

\begin{tabular}{|c|c|c|c|c|c|}
\hline & $E_{\gamma}(\mathrm{eV})$ & FWHM (eV) & $A_{\text {s.i. }}$ & $A_{\text {d.i. }}$ & $A_{\text {t.i. }}$ \\
\hline $\mathrm{P} 1$ & $285.37 \pm 0.52$ & $0.58 \pm 0.02$ & $24.8 \pm 0.9 \%$ & $5.5 \pm 0.2 \%$ & $0.2 \pm 0.0 \%$ \\
\hline $\mathrm{P} 2$ & $287.19 \pm 0.45$ & $2.71 \pm 0.20$ & $22.4 \pm 1.3 \%$ & $4.9 \pm 0.3 \%$ & $0.2 \pm 0.1 \%$ \\
\hline P3 & $290.82 \pm 1.14$ & $* 2.91 \pm 0.30$ & $0.0 \pm 0.0 \%$ & $10.0 \pm 1.2 \%$ & $0.1 \pm 0.1 \%$ \\
\hline P4 & $291.55 \pm 0.21$ & $* 4.37 \pm 0.20$ & $85.2 \pm 2.1 \%$ & $0.0 \pm 0.0 \%$ & $0.0 \pm 0.0 \%$ \\
\hline P5 & $298.56 \pm 0.99$ & $9.72 \pm 1.57$ & $100.0 \pm 2.6 \%$ & $1.4 \pm 2.4 \%$ & $0.3 \pm 0.3 \%$ \\
\hline P6 & $301.77 \pm 0.51$ & $* 6.76 \pm 0.70$ & $0.0 \pm 0.0 \%$ & $25.6 \pm 2.0 \%$ & $1.3 \pm 0.3 \%$ \\
\hline P7 & $305.65 \pm 1.40$ & $3.65 \pm 1.45$ & $3.2 \pm 1.0 \%$ & $3.9 \pm 0.7 \%$ & $0.3 \pm 0.1 \%$ \\
\hline
\end{tabular}

data. Vertical lines (dashed) mark the peak positions (see Table II).

It seems probable that what we perceive as one threshold is in reality composed of several thresholds: For the construction of a suitable basis function representing the necessary threshold step contributions to the observed cross sections, the convolution of a step function with a 4-eV Gaussian was found to be appropriate. While the experimental bandwidth was smaller than $0.25 \mathrm{eV}$, we chose a $0.25-\mathrm{eV}$ Gaussian to conservatively simulate the experimental response function in the fitting procedure. The choice of this width had no significant influence on the results because all features P1-P7 are much broader than the assumed $250 \mathrm{meV}$ bandwidth. To model triple ionization, another very wide feature was needed (dotted line).

\section{Fitting results and findings}

The set of resonances that one can identify in every investigated reaction channel may be explained by resonant excitation of a $K$-shell electron and a subsequent Auger process that occurs when the vacancy in the $K$ shell is filled. An Auger process removing a single electron from the ion is more probable than an Auger process removing two electrons. As a result, these resonance peaks are more intense in single ionization [Fig. 3(a)] than in double ionization [Fig. 3(b)]. In the triple ionization channel [Fig. 3(c)] the intensity is much weaker than in double ionization.

In the double and triple ionization channels, we can observe a threshold at about $294 \mathrm{eV}$ which is not visible for single ionization. This ionization threshold is expected to originate from direct $K$-shell photoionization of one of the carbon atoms. From the fact that there is no visible threshold in single ionization, we can conclude that the probability for the $K$-shell hole to decay via an Auger process is orders of magnitude larger than its probability for a radiative decay: Similar to $K$-vacancy production in a neutral carbon atom, the direct $K$-shell ionization of one atom of the $\mathrm{C}_{80}$ carbon cage almost always removes two or more electrons. Hence, single ionization after $K$-shell photoionization is very unlikely. Photoionization of the $K$ shell releases an energetic electron. Higher order processes can result in the loss of further electrons.
The distribution of area among the peaks (insets of Fig. 3) in channels exhibiting a threshold behavior [namely, double (b) and triple ionization (c) channels] is similar. The area distribution for single ionization (a), however, is strikingly different from (b) and (c). In detail: For double and triple ionization, P6 is dominating by far. P4 is very weak and P5 and P7 just form a low pedestal to P6. However, the three features $\mathrm{P} 1, \mathrm{P} 2$, and $\mathrm{P} 3$ as a group are stronger in double ionization as compared to triple ionization. For single ionization, P3, P6, and P7 are very weak but P4 and P5 are very strong yet similar in intensity. It is common to all channels that $\mathrm{P} 2$ covers a larger area than P1. The peaks P1, P2, and P5 are present in every investigated channel.

The results shown in Fig. 4 were not individually modeled with a fit. Instead, the fitting results for single and double ionization of $\mathrm{Lu}_{3} \mathrm{~N} @ \mathrm{C}_{80}{ }^{+}$from Fig. 3 are plotted together with the corresponding spectra in Fig. 4 after scaling with a constant (energy-independent) factor.

Panels (a) and (b) in Fig. 4 show relative cross sections for single-ionization processes. The overall shape of single ionization of $\mathrm{Lu}_{3} \mathrm{~N} @ \mathrm{C}_{80}{ }^{2+}$ agrees quite well with that of $\mathrm{Lu}_{3} \mathrm{~N} @ \mathrm{C}_{80}{ }^{+}$represented by the fitting result from Fig. 3(a). The data for single ionization of $\mathrm{Lu}_{3} \mathrm{~N} @ \mathrm{C}_{80}{ }^{3+}$ forming $\mathrm{Lu}_{3} \mathrm{~N} @ \mathrm{C}_{80}{ }^{4+}$ [Fig. 4(b)] do not have as good statistics as the preceding channels but overall, the fitting results obtained from Fig. 3(a) for single ionization of $\mathrm{Lu}_{3} \mathrm{~N} @ \mathrm{C}_{80}+$ also fit single ionization of $\mathrm{Lu}_{3} \mathrm{~N} @ \mathrm{C}_{80}{ }^{3+}$.

Figure 4(c) shows a hint on how the spectrum is modified if double ionization of $\mathrm{Lu}_{3} \mathrm{~N} @ \mathrm{C}_{80}{ }^{+}$is accompanied by the loss of a carbon dimer $\mathrm{C}_{2}$. It appears that in the fragmentation channel P1 is stronger in relation to the height of the ionization threshold. Additionally, the fragmentation spectrum slightly deviates from the fitting curve at higher energies.

Figure 4(d) shows relative cross sections for double ionization of $\mathrm{Lu}_{3} \mathrm{~N} @ \mathrm{C}_{80}{ }^{2+}$ together with the fitting result for double ionization of $\mathrm{Lu}_{3} \mathrm{~N} @ \mathrm{C}_{80}{ }^{+}$. This comparison is discussed in more detail below.

\section{Energy shift of the ionization threshold}

When comparing double ionization of the singly charged primary ion [Fig. 3(b)] with double ionization of the doubly charged primary ion [Fig. 4(d)], one finds the ionization 
threshold energy is shifted by $\Delta W=(2.90 \pm 0.24) \mathrm{eV}$. The higher charge of $\mathrm{Lu}_{3} \mathrm{~N} @ \mathrm{C}_{80}{ }^{2+}$ primary ions results in an increase of the attractive Coulomb potential that the fullerene shell of $\mathrm{Lu}_{3} \mathrm{~N} @ \mathrm{C}_{80}{ }^{q+}$ exerts on the electron leaving the fullerene from its surface. Based on the radial Coulomb potential $W_{\text {pot }}$ and the energy shift $\Delta W_{\text {pot }}$ of the threshold, we can provide an estimate for the radius $R$ of the endohedral fullerene $\mathrm{Lu}_{3} \mathrm{~N} @ \mathrm{C}_{80}$ :

$$
\Delta W_{\mathrm{pot}}=\frac{e \Delta q}{4 \pi \epsilon_{0} R},
$$

where $e$ is the elementary charge, $\epsilon_{0}$ the electrical constant, and $\Delta q=2 e-1 e=e$. With the above mentioned energy shift, we find an outer endohedral-fullerene radius of $5.0 \pm 0.4 \times$ $10^{-10} \mathrm{~m}$.

As far as the $K$-shell ionization threshold behavior is concerned, single-ionization cross-section plots all look alike regardless of the initial charge state. In contrast, the discrete ionization threshold present in double and triple ionization is sensitive to the charge state of the primary ion.

\section{E. Comparison with other fullerene species}

Figure 5 compares the photoabsorption of different fullerene species near the carbon $K$ edge with our results. Each cross section was individually scaled such that the highest feature represents $100 \%$.

Panel (d) shows the present photoionization data, whereas panels (a), (b), (c), and (e) show photoabsorption data: (a) data for $\mathrm{C}_{60}$ in the gas phase (black squares) [44] and for a solid (orange squares) [45], (b) results for solid $\mathrm{C}_{70}$ [45], (c) data for solid $\mathrm{C}_{80}$ [46], (d) cross-section data for single ionization (light-gray circles) and double ionization (black squares) of

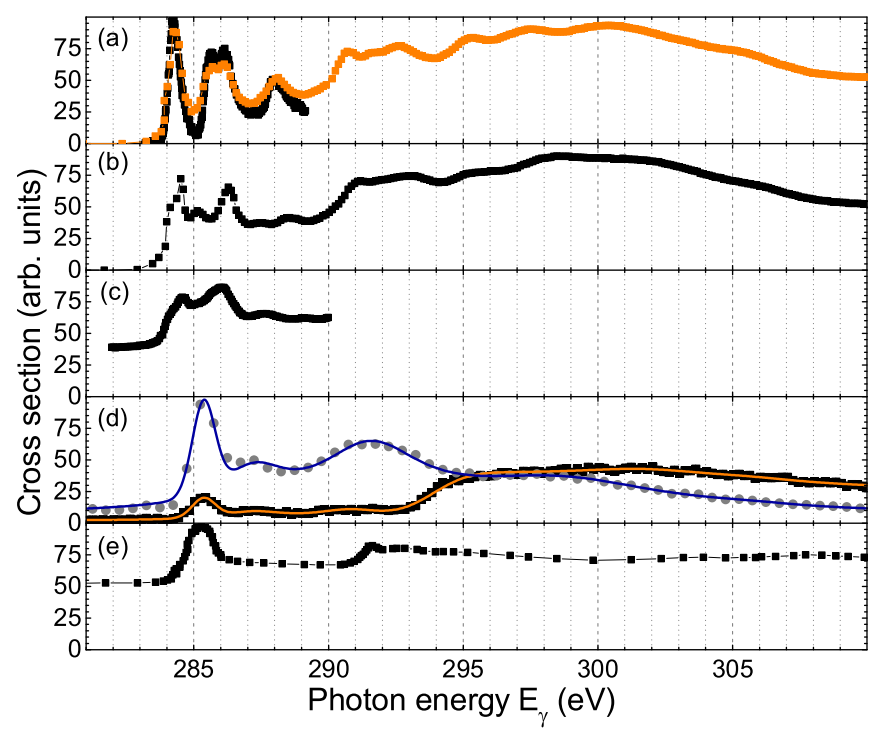

FIG. 5. (Color online) Comparison of photoabsorption of a range of fullerene species around the carbon $K$-shell ionization threshold with cross-section data from the present study. Photoabsorption of (a) $\mathrm{C}_{60}$ in the gas phase (black squares) [44] and solid $\mathrm{C}_{60}$ (orange squares) [45], (b) solid $\mathrm{C}_{70}$ [45], (c) solid $\mathrm{C}_{80}$ [46], and (e) graphite [45]. $\mathrm{Lu}_{3} \mathrm{~N} @ \mathrm{C}_{80}{ }^{+}$cross-section data in panel (d): Single ionization (light-gray circles) and double ionization (black squares).
$\mathrm{Lu}_{3} \mathrm{~N} @ \mathrm{C}_{80}{ }^{+}$. The solid lines in panel (d) represent the fitting results for these channels [see Fig. 3, panels (a) and (b)]. Panel (e) shows data for graphite [45]. Since our datasets are sensitive to individual ionization channels, whereas absorption spectra correspond to the sum of all possible channels for ionization, excitation, etc., a quantitative comparison is meaningless. However, it is surprising that the spectra for the two strongest ionization channels seem to be closer to the absorption plot of graphite than to that of $\mathrm{C}_{80}$.

We find the spectral onsets of $\mathrm{Lu}_{3} \mathrm{~N} @ \mathrm{C}_{80}{ }^{+}$single and double ionization (associated with $K$-shell excitation) at a similar energy. Cummins et al. [46] reported $284.5 \mathrm{eV}$ for $\mathrm{C}_{80}$. We found $285.4 \mathrm{eV}$ for single and double ionization of $\mathrm{Lu}_{3} \mathrm{~N} @ \mathrm{C}_{80}{ }^{+}$. Cummins et al. [46] assign $K$-shell excitations to unoccupied $\pi *$ levels to the $\mathrm{C}_{80}$ features for photon energies $E_{\gamma}>290 \mathrm{eV}$ and excitations into unoccupied $\sigma^{*}$ levels to features with $E_{\gamma}<290 \mathrm{eV}$. Terminello et al. [45] found the same distinction for fullerenes $\left(\mathrm{C}_{60}, \mathrm{C}_{70}\right)$ and graphite based on semiempirical Hückel calculations.

With these considerations in mind, the peak features P1 and $\mathrm{P} 2$ in our data might be assigned to $\sigma^{*}$ transitions and the other resonances (appearing at higher energies) to $\pi^{*}$ transitions. However, with several eV width, features P5 and P6 (possibly also features P4 and P7) are candidates for plasmon excitations [32]. It is difficult to identify features in the absorption spectra corresponding to these wide peaks in the present data. For example, there is no feature like P4 (at $291.5 \mathrm{eV}$ ) visible for graphite. The reduction of the number of resonance features could indicate a change of symmetry of the system. With the available data it is not possible to draw a conclusion on the question if the presence of the encaged molecule reduces the symmetry of $\mathrm{C}_{80}$ so much that the electronic structure has more similarities to bulk graphite than to a $\mathrm{C}_{80}$ molecule.

\section{F. Results for higher photon energies}

Beyond the carbon $K$ edge, we scanned the product channels $\left(\mathrm{Lu}_{3} \mathrm{~N} @ \mathrm{C}_{80}{ }^{+} \rightarrow \mathrm{Lu}_{3} \mathrm{~N} @ \mathrm{C}_{80}{ }^{2+, 3+, 4+}\right)$ at energies from 390 to $435 \mathrm{eV}$ comprising the $K$-shell ionization threshold of the encapsulated nitrogen atom. The measured cross sections show no structures and decrease with increasing photon energies. Therefore, these data are not shown here.

We have also looked for the $M$-shell threshold of lutetium in several product channels: Reactions with a singly charged initial state and final states $\mathrm{Lu}_{3} \mathrm{~N} @ \mathrm{C}_{80}{ }^{3+}, \mathrm{Lu}_{3} \mathrm{~N} @ \mathrm{C}_{80}{ }^{4+}$, $\mathrm{Lu}_{3} \mathrm{~N} @ \mathrm{C}_{80}{ }^{5+}, \mathrm{Lu}_{3} \mathrm{~N} @ \mathrm{C}_{72}{ }^{5+}, \mathrm{Lu}_{3} \mathrm{~N} @ \mathrm{C}_{80}{ }^{6+}, \mathrm{Lu}_{3} \mathrm{~N} @ \mathrm{C}_{74}{ }^{6+}$ have been investigated in the range 1500-1700 eV. Figure 6 shows an overview of the ion yields for the four channels with the lowest statistical uncertainties and a sum of the individual spectra. Similar to the situation near the nitrogen $K$ edge, we found cross sections lacking structures such as resonances or threshold steps.

The channels for finding the lutetium threshold are not exhausted: There are many ionization and/or fragmentation channels left for exploration and it is also possible that the threshold strength is distributed over many different channels including the channels we have already investigated. In consequence, the individual fraction of the total $\mathrm{Lu} M$-shell oscillator strength contributing to an individual final channel 


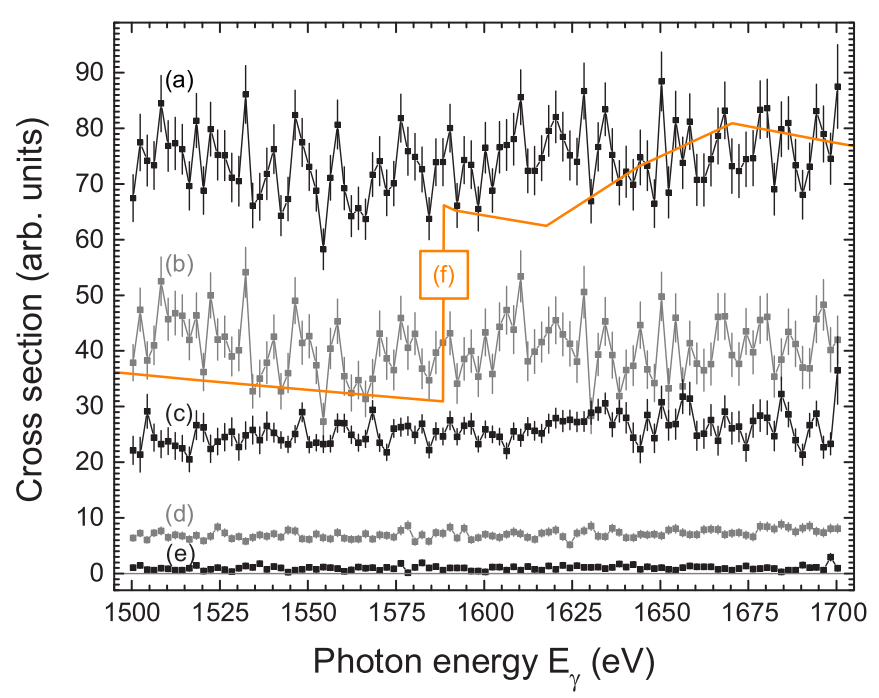

FIG. 6. (Color online) Photoreaction cross-section spectra in the energy range $1500-1700 \mathrm{eV}$ of the $\mathrm{Lu}_{3} \mathrm{~N} @ \mathrm{C}_{80}{ }^{1+}$ initial state. (a) Black symbols: sum of the four individual spectra (b)-(e), (b) gray symbols: $\mathrm{Lu}_{3} \mathrm{~N} @ \mathrm{C}_{80}{ }^{3+}$ product ions, (c) black symbols: $\mathrm{Lu}_{3} \mathrm{~N} @ \mathrm{C}_{80}{ }^{4+}$ product ions, (d) gray symbols: $\mathrm{Lu}_{3} \mathrm{~N} @ \mathrm{C}_{72}{ }^{5+}$ product ions, (e) black symbols: $\mathrm{Lu}_{3} \mathrm{~N} @ \mathrm{C}_{74}{ }^{6+}$ product ions, and (f) solid orange line: prediction for the Lu photoabsorption cross section near the $M$ edge by Henke et al. [47] based on semiempirical calculations.

of fragmentation and ionization of the primary endohedral fullerene might be too small to be easily detected.

\section{SUMMARY AND CONCLUSIONS}

The present results prove that the newly built permanent end station PIPE is suitable for investigations involving weak beams of heavy molecular ions such as $\mathrm{Lu}_{3} \mathrm{~N} @ \mathrm{C}_{80}{ }^{+}$.

The current measurements are part of our endeavor to study photoprocesses of endohedral fullerenes Ce@ $\mathrm{C}_{82}$ [23], $\mathrm{Sc}_{3} \mathrm{~N} @ \mathrm{C}_{80}$ [24], as well as $\mathrm{Xe} @ \mathrm{C}_{60}[28,29,35]$ and extends the range of investigated fullerene species to even heavier molecules encapsulated inside a fullerene.

Strong photoionization signals have been observed for single, double, and triple ionization of $\mathrm{Lu}_{3} \mathrm{~N} @ \mathrm{C}_{80}{ }^{+q}(q=$ $1,2,3$ ) in the energy region of the carbon $K$-shell ionization threshold (280-330 eV). To facilitate quantitative analysis of the spectral features, three prominent ionization channels $\left(\mathrm{Lu}_{3} \mathrm{~N} @ \mathrm{C}_{80}+1 \longrightarrow 2+, 3+, 4+\right)$ have been modeled with a fitting function. A set of resonance peaks is present independently of the primary charge state but with strong variations of the intensity distribution among the peaks from channel to channel. Some of the resonances in the $\mathrm{Lu}_{3} \mathrm{~N} @ \mathrm{C}_{80}{ }^{+}$spectra are very broad, thus indicating possible plasmonic origin.

In contrast, the ionization continuum threshold that is present in the double and triple ionization channels is shifted towards higher energies if the reaction starts from an ion in a higher charge state. Based upon the energy shift of the threshold for direct $K$-shell ionization an estimate for the radius of the almost spherical $\mathrm{Lu}_{3} \mathrm{~N} @ \mathrm{C}_{80}$ molecule could be provided.

As expected, a comparison of our results with absorption spectra of various fullerene species in this energy region shows that the spectral onsets have about the same energy. It appears, however, that photoionization spectra of $\mathrm{Lu}_{3} \mathrm{~N} @ \mathrm{C}_{80}{ }^{+}$have more similarities with the photoabsorption of bulk graphite than with photoabsorption of $\mathrm{C}_{80}$.

While carbon signatures are distinctly present, signals of the atoms encapsulated within the carbon cage could not be identified in the photoion channels presently observed. Several photoreaction channels with fragmented products have been scanned around the energy of the expected lutetium $M$-shell threshold but the cross sections were small and free from structures. This finding leads us to believe that possibly the whole molecular system disintegrates completely if a lutetium atom is excited by one energetic photon: Whenever a lutetium atom is $M$-shell excited, the amount of energy deposited and then released inside the fullerene is so big that the cage falls apart into many small fragments. Since there is a multitude of possible fragmentation channels to which the $\mathrm{Lu} M$-shell oscillator strength is distributed, the lutetium contribution to each individual channel could be very small. Such a triggered radiation damage is of potential interest in various fields of applied research such as radiation biology or material science.

\section{ACKNOWLEDGMENTS}

We thank the Federal State of Hesse, Germany, for financial support through the LOEWE funding scheme (project ELCH). A.M. acknowledges support from Deutsche Forschungsgemeinschaft (DFG) through Grant No. Mu-1068/22-1. Substantial funding from the German Federal Ministry of Education and Research (BMBF) has made construction and building of the PIPE setup possible (Contracts No. 05KS7RG1, No. 05K10RG1, No. 05KS7GU2, and No. 05K10GUB within the Verbundforschung funding scheme). Parts of this research were carried out at the light source PETRA III at DESY, a member of the Helmholtz Association (HGF). We would like to thank J. Viefhaus, L. Glaser, F. Scholz, and J. Seltmann for assistance in using beamline P04.
[1] J. Cioslowski and A. Nanayakkara, Phys. Rev. Lett. 69, 2871 (1992).

[2] L. Becker, R. J. Poreda, A. G. Hunt, T. E. Bunch, and M. Rampino, Science 291, 1530 (2001).

[3] A. Takeda, Y. Yokoyama, S. Ito, T. Miyazaki, H. Shimotani, K. Yakigaya, T. Kakiuchi, H. Sawa, H. Takagi, K. Kitazawa, and N. Dragoe, Chem. Commun. 8, 912 (2006).
[4] J. B. Melanko, M. E. Pearce, and A. K. Salem, in Nanotechnology in Drug Delivery, edited by M. M. Villiers, P. Aramwit, and G. S. Kwon, Biotechnology: Pharmaceutical Aspects Vol. X (Springer, New York, 2009), pp. 105-127.

[5] W. Harneit, C. Boehme, S. Schaefer, K. Huebener, K. Fostiropoulos, and K. Lips, Phys. Rev. Lett. 98, 216601 (2007).

[6] M. J. Puska and R. M. Nieminen, Phys. Rev. A 47, 1181 (1993). 
[7] J. P. Connerade, V. K. Dolmatov, and S. T. Manson, J. Phys. B 33, 2279 (2000).

[8] H. W. Kroto, J. R. Heath, S. C. O'Brien, R. F. Curl, and R. E. Smalley, Nature (London) 318, 162 (1985).

[9] J. R. Heath, S. C. O'Brien, Q. Zhang, Y. Liu, R. F. Curl, F. K. Tittel, and R. E. Smalley, J. Am. Chem. Soc. 107, 7779 (1985).

[10] Y. Chai, T. Guo, C. Jin, R. E. Haufler, L. P. F. Chibante, J. Fure, L. Wang, J. M. Alford, and R. E. Smalley, J. Phys. Chem. 95, 7564 (1991).

[11] M. J. Puska and R. M. Nieminen, Phys. Rev. A 49, 629 (1994).

[12] M. Y. Amusia, A. S. Baltenkov, L. V. Chernysheva, Z. Felfli, and A. Z. Msezane, J. Phys. B 38, L169 (2005).

[13] M. Y. Amusia, J. Electron Spectrosc. Relat. Phenom. 161, 112 (2007).

[14] M. Y. Amusia, A. S. Baltenkov, and L. V. Chernysheva, Phys. Rev. A 75, 043201 (2007).

[15] M. Amusia, A. Baltenkov, and L. Chernysheva, JETP Lett. 87, 200 (2008).

[16] M. Y. Amusia, L. V. Chernysheva, and E. Z. Liverts, Phys. Rev. A 80, 032503 (2009).

[17] M. E. Madjet, T. Renger, D. E. Hopper, M. A. McCune, H. S. Chakraborty, Jan-M. Rost, and S. T. Manson, Phys. Rev. A 81, 013202 (2010).

[18] Z. Chen and A. Z. Msezane, J. Phys. B 45, 235205 (2012).

[19] T. W. Gorczyca, M. F. Hasoglu, and S. T. Manson, Phys. Rev. A 86, 033204 (2012).

[20] B. Li, G. O’Sullivan, and C. Dong, J. Phys. B 46, 155203 (2013).

[21] K. Mitsuke, T. Mori, J. Kou, Y. Haruyama, and Y. Kubozono, J. Chem. Phys. 122, 064304 (2005).

[22] K. Mitsuke, T. Mori, J. Kou, Y. Haruyama, Y. Takabayashi, and Y. Kubozono, Int. J. Mass Spectrom. 243, 121 (2005).

[23] A. Müller, S. Schippers, M. Habibi, D. Esteves, J. C. Wang, R. A. Phaneuf, A. L. D. Kilcoyne, A. Aguilar, and L. Dunsch, Phys. Rev. Lett. 101, 133001 (2008).

[24] A. Müller, S. Schippers, R. A. Phaneuf, M. Habibi, D. Esteves, J. C. Wang, A. L. D. Kilcoyne, A. Aguilar, S. Yang, and L. Dunsch, J. Phys.: Conf. Ser. 88, 012038 (2007).

[25] A. Müller, S. Schippers, R. A. Phaneuf, S. Scully, E. D. Emmons, M. F. Gharaibeh, M. Habibi, A. L. D. Kilcoyne, A. Aguilar, A. S. Schlachter, L. Dunsch, S. Yang, H. S. Chakraborty, M. E. Madjet, and J. M. Rost, in Latest Advances in Atomic Cluster Collisions: Structure and Dynamics from the Nuclear to the Biological Scale, edited by J.-P. Connerade and A. V. Solov'yov (Imperial College Press, London, UK, 2008), pp. 177-186.

[26] A. Müller, S. Schippers, D. Esteves, M. Habibi, R. A. Phaneuf, A. L. D. Kilcoyne, A. Aguilar, and L. Dunsch, in The Forth International Symposium on Atomic Cluster Collisions (ISACC 2009), AIP Conf. Proc. No. 1197 (AIP, Melville, NY, 2009), pp. 103-110.

[27] A. V. Korol and A. V. Solov'yov, J. Phys. B 43, 201004 (2010).

[28] A. L. D. Kilcoyne, A. Aguilar, A. Müller, S. Schippers, C. Cisneros, G. Alna'Washi, N. B. Aryal, K. K. Baral, D. A. Esteves, C. M. Thomas, and R. A. Phaneuf, Phys. Rev. Lett. 105, 213001 (2010).

[29] R. A. Phaneuf, A. L. D. Kilcoyne, N. B. Aryal, K. K. Baral, D. A. Esteves-Macaluso, C. M. Thomas, J. Hellhund, R. Lomsadze, T. W. Gorczyca, C. P. Ballance, S. T. Manson, M. F. Hasoglu, S. Schippers, and A. Müller, Phys. Rev. A 88, 053402 (2013).
[30] S. Schippers, S. Ricz, T. Buhr, A. Borovik, Jr., J. Hellhund, K. Holste, K. Huber, H.-J. Schäfer, D. Schury, S. Klumpp, K. Mertens, M. Martins, R. Flesch, G. Ulrich, E. Rühl, T. Jahnke, J. Lower, D. Metz, L. P. H. Schmidt, M. Schöffler et al., J. Phys. B 47, 115602 (2014).

[31] J. Viefhaus, F. Scholz, S. Deinert, L. Glaser, M. Ilchen, J. Seltmann, P. Walter, and F. Siewert, Nucl. Instrum. Methods A 710, 151 (2013).

[32] S. W. J. Scully, E. D. Emmons, M. F. Gharaibeh, R. A. Phaneuf, A. L. D. Kilcoyne, A. S. Schlachter, S. Schippers, A. Müller, H. S. Chakraborty, M. E. Madjet, and J. M. Rost, Phys. Rev. Lett. 94, 065503 (2005).

[33] S. W. J. Scully, E. D. Emmons, M. F. Gharaibeh, R. A. Phaneuf, A. L. D. Kilcoyne, A. S. Schlachter, S. Schippers, A. Müller, H. S. Chakraborty, M. E. Madjet, and J. M. Rost, Phys. Rev. Lett. 98, 179602 (2007).

[34] R. C. Bilodeau, N. D. Gibson, C. W. Walter, D. A. EstevesMacaluso, S. Schippers, A. Müller, R. A. Phaneuf, A. Aguilar, M. Hoener, J. M. Rost, and N. Berrah, Phys. Rev. Lett. 111, 043003 (2013).

[35] R. A. Phaneuf, A. L. D. Kilcoyne, A. Müller, S. Schippers, N. Aryal, K. Baral, J. Hellhund, A. Aguilar, D. A. EstevesMacaluso, and R. Lomsadze, in Eighth International Conference on Atomic and Molecular Data and Their Applications, AIP Conf. Proc. No. 1545 (AIP, Melville, NY, 2013), p. 72.

[36] R. B. Ross, C. M. Cardona, D. M. Guldi, S. G. Sankaranarayanan, M. O. Reese, N. Kopidakis, J. Peet, B. Walker, G. C. Bazan, E. V. Keuren, B. C. Holloway, and M. Drees, Nat. Mater. 8, 208 (2009).

[37] F. H. Hennrich, R. H. Michel, A. Fischer, S. Richard-Schneider, S. Gilb, M. M. Kappes, D. Fuchs, M. Bürk, K. Kobayashi, and S. Nagase, Angew. Chem. 108, 1839 (1996).

[38] R. Trassl, in The Physics of Multiply and Highly Charged Ions, edited by F. Currell (Kluwer Academic, Dordrecht, 2003), Vol. 1, pp. 3-37.

[39] J. Fricke, A. Müller, and E. Salzborn, Nucl. Instrum. Methods 175, 379 (1980).

[40] K. Rinn, A. Müller, H. Eichenauer, and E. Salzborn, Rev. Sci. Instrum. 53, 829 (1982).

[41] A. S. Schlachter, M. M. Sant'Anna, A. M. Covington, A. Aguilar, M. F. Gharaibeh, E. D. Emmons, S. W. J. Scully, R. A. Phaneuf, G. Hinojosa, I. Álvarez, C. Cisneros, A. Müller, and B. M. McLaughlin, J. Phys. B 37, L103 (2004).

[42] A. Müller, S. Schippers, R. A. Phaneuf, S. W. J. Scully, A. Aguilar, A. M. Covington, I. Álvarez, C. Cisneros, E. D. Emmons, M. F. Gharaibeh, G. Hinojosa, A. S. Schlachter, and B. M. McLaughlin, J. Phys. B 42, 235602 (2009).

[43] M. Oura, Plasma Sci. Technol. 12, 353 (2010).

[44] S. Krummacher, M. Biermann, M. Neeb, A. Liebsch, and W. Eberhardt, Phys. Rev. B 48, 8424 (1993).

[45] L. Terminello, D. Shuh, F. Himpsel, D. Lapiano-Smith, J. Stöhr, D. Bethune, and G. Meijer, Chem. Phys. Lett. 182, 491 (1991).

[46] T. R. Cummins, M. Bürk, M. Schmidt, J. F. Armbruster, D. Fuchs, P. Adelmann, S. Schuppler, R. H. Michel, and M. M. Kappes, Chem. Phys. Lett. 261, 228 (1996).

[47] B. Henke, E. Gullikson, and J. Davis, At. Data Nucl. Data Tables 54, 181 (1993). 